\title{
WFirSt Ultra-Precise Astrometry I: Kuiper Belt ObJects
}

\author{
ANDREW GOULD \\ Department of Astronomy, Ohio State University, 140 W. 18th Ave., Columbus, OH 43210, USA \\ gould@astronomy . ohio-state.edu
}

Received August 4, 2014; accepted November 20, 2014

\begin{abstract}
I show that the WFIRST microlensing survey will enable detection and precision orbit determination of Kuiper Belt Objects (KBOs) down to $H_{\text {vega }}=28.2$ over an effective area of $\sim 17 \mathrm{deg}^{2}$. Typical fractional period errors will be $\sim 1.5 \% \times 10^{0.4(H-28.2)}$ with similar errors in other parameters for roughly $5000 \mathrm{KBOs}$. Binary companions to detected KBOs can be detected to even fainter limits, $H_{\text {vega }}=29$, corresponding to $R \sim 30.5$ and effective diameters $D \sim 7 \mathrm{~km}$. For KBOs $H \sim 23$, binary companions can be found with separations down to 10 mas. This will provide an unprecedented probe of orbital resonance and KBO mass measurements. More than a thousand stellar occultations by KBOs can be combined to determine the mean size as a function of KBO magnitude down to $H \sim 25$. Current ground-based microlensing surveys can make a significant start on finding and characterizing KBOs using existing and soon-to-be-acquired data.
\end{abstract}

Key words: astrometry — Kuiper belt — gravitational microlensing

\section{INTRODUCTION}

Kuiper Belt Objects (KBOs) provide an extraordinary probe of the origin and history of the Solar System. When Pluto was discovered by Clyde Tombaugh (Slipher 1930a,b) and was then found to be in a 3:2 resonance with Neptune, it was hardly guessed that it was only the largest of a vast class of such objects. Subsequent discovery of KBOs in 2:1 resonance, in various kinematic and composition subclasses, of binary KBOs, and of a break in the size distribution at $R \sim 26.5$ (Bernstein et al. 2004) have placed extremely detailed constraints on early Solar System evolution, even leading to radical conjectures like the idea that Uranus and Neptune originally formed much closer to the orbits of Jupiter and Saturn (Gomes et al. 2005). I show that the WFIRST microlensing survey will, without any adjustment, yield a KBO survey that is both substantially deeper and three orders of magnitude wider and more precise than existing deep KBO surveys based on $H u b$ ble Space Telescope (HST) data.

\section{KBOs in Microlensing DATA}

Microlensing surveys are conducted toward the densest star fields on the sky and therefore would appear at first sight to be the worst place to carry out a search for KBOs. In particular, confusion in the identification of moving objects is known to be an extremely strong function of the density of the stellar background (e.g., Monet et al. 2003).

Surprisingly, microlensing fields are actually the best place to conduct deep searches for moving objects, KBOs in particular. First, contrary to naive expectation, there is no problem of confusion at all. Microlensing searches are conducted on a series of differ-

Corresponding Author: A. Gould ence images, which are constructed by first forming a deep (essentially noiseless) reference image from many individual images, aligning each image geometrically to the reference image, convolving the reference image to the point spread function (PSF) of each image, aligning each image photometrically to the reference image, and finally subtracting each image from the reference image (Alard \& Lupton 1998). The result is a virtually flat image with essentially all stars removed and only photon and detector noise left. The only exceptions are stars (or other objects) that have changed relative to the reference image, either changed their brightness or changed their position, and a very small number of artifacts due to difficulties in subtracting extremely bright and/or saturated stars. Hence, microlensing fields are not crowded at all: they are essentially blank, much more so than high-latitude fields.

Second, the noise properties of every pixel (as a function of both position on the sky and position within the detector array) are understood essentially perfectly. This is because there are hundreds, thousands, or even tens of thousands of images of the same field. In the case of ground-based surveys, these encompass the full range of observing conditions, while for space-based surveys, the images are taken under constant conditions (or rather conditions that vary much less than is relevant to faint objects).

Third, microlensing surveys carry out hundreds or thousands of observations during a single season (during which KBOs remain mostly within the microlensing fields). While each individual exposure is not particularly deep, the fact that the images are essentially blank means it is straightforward to combine many observations to detect faint KBOs. In particular, while the KBO will occasionally "land" at the position of a relatively bright star, the precise knowledge of high noise 
at this location (derived from photometric deviations on hundreds of other images - see previous paragraph) will automatically "suppress" this observation within the ensemble. That is, KBO searches will automatically be dominated by the highest signal-to-noise ratio (SNR) observations.

Fourth, because the Galactic Center is only about $6^{\circ}$ south of the ecliptic, microlensing surveys automatically probe regions of the sky on and near the ecliptic.

Finally, the high density of stellar background is actually an advantage because it gives rise to occultations that can be used to measure the size distribution.

To date, no one has yet suggested, let alone explored, the possibility of KBO searches in microlensing fields. Here, I focus on the potential of future space based surveys, but the same principle applies to ground-based data. In particular, the Optical Gravitational Lens Experiment (OGLE) survey already has more than 4 years of data covering about $5 \mathrm{deg}^{2}$ with a cadence of roughly 30 observations per night during peak microlensing season, with typical seeing slightly more that $1^{\prime \prime}$, while the new Korean Microlensing Telescope Network (KMTNet) survey is expected to cover $16 \mathrm{deg}^{2}$ with a cadence of about 120 observations per night with only slightly worse seeing (counting only Chile and South Africa telescopes).

While no KBO searches have been conducted using microlensing data, OGLE did search for KBOs in the $2500 \mathrm{deg}^{2}$ of the southern Galactic plane (Sheppard et al. 2011). Because there were only three exposures, each $180 \mathrm{~s}$ (for the overwhelming majority of fields), the survey reached only $R=21.6$. Highly illustrative is their "rediscovery" of Pluto at roughly $(l, b)=(13,-2)$ in a dense stellar field (their Figure 3), which becomes essentially blank (except for Pluto) in the difference image (their Figure 4).

\section{Astrometry From A Microlensing Survey}

A space-based microlensing survey will almost automatically return high-quality astrometric data. I discuss this potential within the framework of the proposed WFIRST (Spergel et al. 2013a,b) survey, but the same principles could be applied to any mission of this type. I adopt the following parameters for the microlensing component of the proposed WFIRST mission.

1) 10 contiguous, $0.28 \mathrm{deg}^{2}$ Galactic bulge fields

2) $\operatorname{six} \Delta t=72$ day continuous "campaigns" , each centered at quadrature (March or September)

3) 15 min cycle time consisting of ten $52 \mathrm{~s}$ exposures

4) $90 \%$ of exposures in broad $H$ band, $10 \%$ in a narrower band, e.g., $Y$

5) $2.4 \mathrm{~m}$ telescope, $\theta_{\text {pix }}=110$ mas pixels,

6 ) one photon per second at $H_{\text {vega }}=26.1$

7) $B=341$ total "counts" per pixel in read noise, zodiacal light and dark current per 52s integration

The diffraction limited point spread function (PSF) has $\mathrm{FWHM}=275$ mas, implying an equivalent Gaussian width $\sigma_{\mathrm{psf}}=\mathrm{FWHM} / 2.35=75$ mas. Due to the slightly undersampled PSF, I assume a total background light of $9 B$, i.e., about 1.5 times larger than the $4 \pi\left(\sigma_{\mathrm{psf}} / p\right)^{2} B$ appropriate to the oversampled limit. This leads to an equivalent "sky" of $H_{\text {sky }}=26.1-2.5 *$ $\log (9 B / 52)=21.7$. Since this paper will concern only sources well below this limit, I restrict further consideration to "below sky" sources. For these, the signalto-noise ratio (SNR) is given by

$$
\mathrm{SNR}=10^{0.4\left(H_{\text {zero }}-H\right)} ; \quad H_{\text {zero }}=26.1
$$

The 15 min cadence implies a total of 6908 observations during a 72 day campaign. Of these $10 \%$ are not in the wide band and so contribute much less astrometric information. These will simply be ignored (though they will provide important color information). I further assume that $10 \%$ of the remainder land on sources that are significantly above sky and therefore have substantial additional noise in the difference images. This leaves

$$
N_{\text {cam }}=5600
$$

images, distributed roughly uniformly in time over each campaign. I assume that each of these has astrometric precision

$$
\sigma_{\mathrm{ast}}=\sqrt{2} \frac{\sigma_{\mathrm{psf}}}{\mathrm{SNR}}=\frac{106 \mathrm{mas}}{\mathrm{SNR}} .
$$

The images will be dithered and the KBOs will move relative to the stellar background, so the astrometric and photometric errors of the $N_{\text {cam }}=5600$ images will be essentially uncorrelated. Because of the very large number of images, the central limit theorem therefore implies that Gaussian statistics strictly apply.

\section{World CoOrdinate System}

In order to fit $\mathrm{KBO}$ orbits over the $\sim 1^{\circ}$ trajectories that they traverse during a 72-day campaign, the WFIRST astrometric frame must be calibrated to at least the precision of the KBO measurements. Since GAIA will measure positions, parallaxes, and proper motions for several million stars in the WFIRST microlensing fields, it appears at first sight that this issue will be taken care of "automatically". While this ultimately proves to be the case, there are some subtleties, which I now address.

The central difficulty is that WFIRST will "saturate" (see below) at $H_{\text {vega }} \sim 14$, while the majority of GAIA stars will be bulge sub-giants and giants with $(V-H)_{0}>1.5$ that lie behind most of the dust with $E(V-H) \sim 3.5$. For such stars to avoid saturation in WFIRST, they must be $G>19$ in the GAIA band (which is near $V$ ). While GAIA will observe stars to significantly fainter magnitudes over most of the sky, it will have a substantially brighter limit in the bulge due to data-rate limitations. Hence, it is not immediately obvious that the WFIRST frame can be tied to the GAIA frame.

The best frame-tying stars are foreground $\mathrm{G}$ dwarfs, with $(V-H)_{0} \sim 1.5$ and $M_{V} \sim 5$. I assume $A_{V}=$ $0.7 \mathrm{mag} \mathrm{kpc}^{-1}$ and $A_{V} / A_{H}=6$. Such stars reach the 
WFIRST saturation limit at $D \sim 1.2 \mathrm{kpc}$ at which $H \sim 14$ and $V \sim 16.2$, while at $D \sim 1.8 \mathrm{kpc}$, the corresponding numbers are $H \sim 15$ and $V \sim 17.5$. These stars will have GAIA $G$ band magnitudes $16.1<G<$ 17.2 and hence GAIA position, parallax, and proper motion precisions ${ }^{\mathbf{1}}$ ranging from $\left(32,43,22 \mathrm{yr}^{-1}\right) \mu$ as to $\left(54,73,39 \mathrm{yr}^{-1}\right) \mu$ as.

To set up a reference frame, all we are really concerned with is how well the positions of these stars will be known roughly $T=10 \mathrm{yr}$ after the midpoint of the GAIA mission when WFIRST is launched. (WFIRST proper motions of these overlap stars will be measured far better than those of GAIA, so we are concerned with overlap at the beginning of the WFIRST mission, not its midpoint.) Hence, the positions will be known to a precision of $200-400 \mu$ as.

The surface density of such stars on the sky is $N=$ $n\left(D_{\max }^{3}-D_{\min }^{3}\right) / 3=4100 \mathrm{deg}^{-2}$, where $n=0.01 \mathrm{pc}^{-3}$ is the number density of $\mathrm{G}$ dwarfs at the mean distance.

These overlap stars must be used for two types of alignment. First, the 10 WFIRST fields must be aligned with each other. Second, the "pixel frame" of the camera must be calibrated to an externally determined angular scale. Since each frame has $0.28 \times 4100 \sim$ 1150 stars, it can be aligned to the global system to $300 \mu \mathrm{as} / \sqrt{1150} \sim 9 \mu \mathrm{as}$. For purposes of internal alignment, all the overlap stars observed in the 10 fields can be used, implying a density of $\sim 41000 \mathrm{deg}^{-2}=$ $\left(18^{\prime \prime}\right)^{-2}$.

At what point do each of these two calibration issues become the limiting factor? Each KBO spends roughly 25 days (or 1800 observations) inside a field, which implies that a $9 \mu$ as calibration error becomes an issue when the individual astrometric error falls below $9 \mu$ as $\sqrt{1800}=380 \mu$ as. This occurs at SNR $=280$, i.e., $H=20$, far brighter than any KBO of practical interest. Similarly, the KBO remains in the neighborhood of a calibration star for $18^{\prime \prime}$, corresponding to about 25 observations, so calibration would become an issue at $\mathrm{SNR}=60$ corresponding to $H=21.7$, still much too bright to be of concern.

I note that WFIRST microlensing fields will be dithered, so that 40,000 images of each field will permit high-precision calibration of the pixel scale on the $18^{\prime \prime}$ scale of the typical separations between calibrating stars.

Finally, I return to the question of the WFIRST saturation limit. The arrays go non-linear at about half the full well of 100,000 photo-electrons. In the broad $H$ filter no more than $30 \%$ of photons land in a single pixel. The pixels are read out every $2.6 \mathrm{~s}$. These numbers lead to a conservative saturation estimate of $H=26.1-2.5 \log (50000 /(0.3 * 2.6)=14.1$. The question of what is "saturated" depends strongly on the application. If one is interested in precise individual photometric measurements, then probably several reads are required, not just the single read in the above calculation. However, if one is interested solely in determining the astrometric center of the PSF to, say, $1 \%$ of

\footnotetext{
${ }^{1}$ http://www. cosmos.esa. int/web/gaia/science-performance
}

a pixel, it is doubtful whether it is actually necessary that the central pixel be unsaturated, let alone in the linear regime, since the position can be centroided from other pixels. Further, one could restrict attention to the subset of observations that land near the corner of four pixels. Thus, it is quite plausible that the saturation limit for astrometry could go 1 mag or more brighter than $H=14$. However, the above calculation shows that for present purposes, such a detailed investigation is unnecessary.

\section{Precision of Orbit Determination}

Assuming that a KBO is identified in the data, how well can its orbit be measured? To answer this question, we must first ask how long it will stay within the field of view. The first point is that at semi-major $a \sim 40 \mathrm{AU}$, and hence period $P=(a / \mathrm{AU})^{3 / 2} \mathrm{yr} \sim 250 \mathrm{yr}$, a KBO will move about $1.4 \mathrm{deg}$ from one year to the next, and hence will typically not return to the microlensing fields the next year. On the other hand, since the fields are observed at quadrature when Earth is accelerating transverse to the line of sight at $A_{\oplus}=4 \pi^{2} \mathrm{AU} \mathrm{yr}^{-2} \sim$ $0.5 \mathrm{~km} \mathrm{~s}^{-1} \mathrm{day}^{-1}$ while the KBO will be moving of order $v_{\perp} \sim 5 \mathrm{kms}^{-1}$, the net projected relative motion of Earth against the KBO will be

$$
\Delta x \sim \sin \left(\frac{2 \pi\left(\Delta t / 2+v_{\perp} / \mathrm{AU}\right)}{\mathrm{yr}}\right) \mathrm{AU} \sim 0.71 \mathrm{AU}
$$

which corresponds to $1.0 \mathrm{deg}$. Hence, of order $60 \%$ of the KBOs will remain in the field for the duration of the campaign. I will initially restrict attention to this subgroup and reserve discussion regarding the remainder to Section 10.

To estimate the precision of the orbital parameters, I approximate Earth as being in a circular orbit and approximate the KBO physical motion during the period of observation as uniform. The deviations from both assumptions are slight and, what is more important, deterministic with respect to the adopted parameters. For example, the acceleration of the KBO is given directly by its distance and position on the sky. Thus, making these assumptions only slightly changes, but vastly simplifies the "trial functions" and hence renders tractable the error estimates while not significantly impacting these estimates. I follow Gould \& Yee (2013) in making the initial estimates in Cartesian phase-space coordinates (instantaneous positions and velocities) rather than the traditional orbital invariants. Of course, actual fits to data will use Kepler invariants, but the Cartesian approach is more closely matched to short timescale observations and therefore facilitates both deeper understanding and simpler results. The implications for Kepler invariants are then easily derived.

The KBO then has motion $\mathbf{x}(t)=\mathbf{x}_{0}+\mathbf{v} t$, described by six parameters $\left(\mathbf{x}_{0}, \mathbf{v}\right)=\left(r_{0}, \mathbf{x}_{\perp}, v_{r}, \mathbf{v}_{\perp}\right)$. I adopt the midpoint of the campaign as the origin of time. For convenience, these six parameters can be re-expressed 
as $\left(\Pi, \boldsymbol{\theta}_{0}, \boldsymbol{\nu}_{\perp}, \nu_{r}\right)$,

$$
\boldsymbol{\theta}_{0} \equiv \frac{\mathbf{x}}{r_{0}} ; \quad \boldsymbol{\nu}_{\perp} \equiv \frac{\mathbf{v}_{\perp}}{\Omega r_{0}} ; \quad \Pi \equiv \frac{\mathrm{AU}}{r_{0}} ; \quad \nu_{r} \equiv \frac{v_{r}}{\Omega r_{0}} .
$$

where $\Omega=2 \pi \mathrm{yr}^{-1}$ is Earth's orbital frequency. The $W F I R S T$ fields are only a few degrees from the ecliptic, and for simplicity I assume that the KBO is directly on the ecliptic.

The first four of these parameters in Equation (5) are essentially direct observables, i.e., the position and instantaneous (normalized) proper motion of the KBO at the zero point of time. The last two pose the main challenge. Since these are derived entirely from the motion of the KBO within the ecliptic plane, I restrict attention to these two dimensions (radial and 1-D transverse). Then the equation for the angular position $\theta(t)$ is

$$
\begin{aligned}
\theta(t) & =\frac{x_{0}+v_{\perp} t-\mathrm{AU}(\cos \Omega t-1)}{r+v_{r} t-\mathrm{AU} \sin \Omega t} \\
& =\frac{\theta_{0}+\nu_{\perp} \Omega t+2 \Pi \sin ^{2}(\Omega t / 2)}{1+\nu_{r} \Omega t-\Pi \sin \Omega t} .
\end{aligned}
$$

Since there are four parameters to be determined, we should expand to third order in time

$$
\theta(t)=\sum_{i=0}^{3} a_{i}(\Omega t)^{i}
$$

where

$$
\begin{aligned}
a_{0}= & \theta_{0} \quad a_{1}=\nu_{\perp}+\theta_{0} Z \quad a_{2}=0.5 \Pi+\nu_{\perp} Z+\theta_{0} Z^{2} \\
a_{3}= & -0.5 \Pi \nu_{r}+0.5 \Pi^{2}+\Pi^{2} \nu_{\perp}-2 \Pi \nu_{\perp} \nu_{r} \\
& +\nu_{r}^{2} \nu_{\perp}+\theta_{0}\left(Z^{3}-\Pi / 6\right)
\end{aligned}
$$

and $Z \equiv \Pi-\nu_{r}$. To a good approximation the four coefficients are well represented by their leading terms

$$
a_{0}=\theta_{0} \quad a_{1} \rightarrow \nu_{\perp} \quad a_{2} \rightarrow 0.5 \Pi \quad a_{3} \rightarrow-0.5 \Pi \nu_{r} .
$$

For a uniform set of $N$ observations over time $\Delta t$, the covariance matrix for these four coefficients is given by (e.g., Gould 2003),

$$
\begin{aligned}
c_{i j}= & \frac{\sigma_{\text {ast }}^{2}}{N_{\text {cam }}}(\Omega \Delta t)^{-(i+j)} \tilde{c}_{i j} \\
\tilde{c}_{i j}= & \left(\begin{array}{cccc}
9 / 4 & 0 & -15 & 0 \\
0 & 75 & 0 & -420 \\
-15 & 0 & 180 & 0 \\
0 & -420 & 0 & 2800
\end{array}\right) .
\end{aligned}
$$

Therefore, the errors in the three angular variables of interest (i.e., excluding $\theta_{0}$ ) are

$$
\begin{aligned}
\frac{\sigma\left(\nu_{\perp}\right)}{\Pi^{3 / 2}} & =\sqrt{\frac{75}{N}}(\Omega \Delta t)^{-1} \Pi^{-3 / 2} \sigma_{\text {ast }}, \\
\frac{\sigma(\Pi)}{\Pi} & =\sqrt{\frac{720}{N}}(\Omega \Delta t)^{-2} \Pi^{-1} \sigma_{\text {ast }}, \\
\frac{\sigma\left(\nu_{r}\right)}{\Pi^{3 / 2}} & =\sqrt{\frac{11200}{N}}(\Omega \Delta t)^{-3} \Pi^{-5 / 2} \sigma_{\text {ast }},
\end{aligned}
$$

where in each case I have normalized to a relevant physical scale. For typical parameters $(\Omega \Delta t \sim 1.25$, $\Pi \sim 1 / 40$ ), the final expression in Equation (11) is larger than either of the others by a factor $>300$. This is the justification for ignoring the correlations between these levels embedded in Equation (8) and simply using Equation (9): the errors in $\nu_{r}$ completely dominate. Translating to physical variables, we obtain

$$
\begin{aligned}
& \frac{\sigma\left(v_{\perp}\right)}{v_{\oplus} \Pi^{1 / 2}}=\sqrt{\frac{75}{N}}(\Omega \Delta t)^{-1} \Pi^{-3 / 2} \sigma_{\text {ast }} \\
\rightarrow & \frac{1.2 \times 10^{-5}}{\mathrm{SNR}}\left(\frac{N}{5600}\right)^{-1 / 2}\left(\frac{\Delta t}{72 \mathrm{~d}}\right)^{-1}\left(\frac{r}{40 \mathrm{AU}}\right)^{3 / 2}, \\
& \frac{\sigma(r)}{r}=\sqrt{\frac{720}{N}}(\Omega \Delta t)^{-2} \Pi^{-1} \sigma_{\text {ast }} \\
\rightarrow & \frac{4.8 \times 10^{-6}}{\mathrm{SNR}}\left(\frac{N}{5600}\right)^{-1 / 2}\left(\frac{\Delta t}{72 \mathrm{~d}}\right)^{-2}\left(\frac{r}{40 \mathrm{AU}}\right), \\
& \frac{\sigma\left(v_{r}\right)}{v_{\oplus} \Pi^{1 / 2}}=\sqrt{\frac{11200}{N}}(\Omega \Delta t)^{-3} \Pi^{-5 / 2} \sigma_{\mathrm{ast}} \\
\rightarrow & \frac{3.8 \times 10^{-3}}{\mathrm{SNR}}\left(\frac{N}{5600}\right)^{-1 / 2}\left(\frac{\Delta t}{72 \mathrm{~d}}\right)^{-3}\left(\frac{r}{40 \mathrm{AU}}\right)^{5 / 2} .
\end{aligned}
$$

These results imply that the orbit-parameter error ellipsoid is essentially a 1-dimensional structure. In Cartesian space, this one dimension is associated with a single parameter: $v_{r}$. After transforming to Kepler coordinates, all parameters inherit this error in $v_{r}$ but in a highly correlated way. For example, for roughly circular orbits, the period error $\sigma(P)$ is

$$
\frac{\sigma(P)}{P}=\frac{3}{2} \frac{\sigma(a)}{a} \simeq \frac{3}{2} \frac{\sigma(\mathrm{KE})}{\mathrm{KE}} \simeq \frac{3 v_{r}}{v_{\oplus} \Pi^{1 / 2}} \frac{\sigma\left(v_{r}\right)}{v_{\oplus} \Pi^{1 / 2}}
$$

where KE is the kinetic energy. Hence, for typical values $v_{r} \sim 0.2 v_{\oplus} \Pi^{1 / 2}$, the fractional period error is somewhat smaller than the last expression in Equation (12). In the next section, I will show that the theoretical limit for finding KBOs is near SNR $\sim 1 / 7$. Thus, even at this limit, the period precision is of the order of $1.5 \%$. At the break in the KBO luminosity function, $R \sim 26.5$, so roughly ${ }^{2} H \sim 25.1$ or $\mathrm{SNR} \sim 2.5$, the period error is $\sigma(P) / P \sim 0.09 \%$.

\footnotetext{
${ }^{2}$ When making comparisons to optical measurements, I adopt $R-H=1.4$, which is about $0.3 \mathrm{mag}$ redder than the Sun. Doressoundiram et al. (2008) (Figures 2 and 3) show that there is a clustering of KBOs near $(V-I, J-H) \sim(0.95,0.35)$, which when compared to the solar values $\sim(0.68,0.32)$, indicates redder optical colors but similar infrared colors to the Sun. However, their Figure 2 also shows that the overall optical color distribution is asymmetric, with a second peak in the distribution, roughly 0.35 to the red in $(V-I)$. My adopted value $R-H=1.4$, which is meant only to give an indication of more commonly used optical magnitudes, is representative of these populations. Note, however, at least some KBOs have optical-infrared colors very similar to the Sun. For example Orcus has $V-J=1.08 \pm 0.04$, compared to $(V-J) \odot=1.08$.
} 


\section{Finding KBOS in the DATA}

WFIRST may well be in geosynchronous orbit. In principle, this would add information to the measurements of $r$ and $v_{r}$. However, as I show below, this added information plays an insignificant role except in the margins of parameter space and was therefore ignored in Section 5. A geosynchronous orbit would also somewhat complicate the search for KBOs in the data and so cannot be completely ignored in the present section. Nevertheless, I begin by ignoring it, partly to cover the case of non-geosynchronous (e.g., L2) orbit and partly to be able to show explicitly, further below, that the complications induced by geosynchronous orbit are not in fact significant.

The WFIRST field has $N_{\text {pix }}=2.8 \mathrm{deg}^{2} /(110 \text { mas })^{2}=$ $3.0 \times 10^{9}$ pixels. Thus, for KBOs with

$$
\mathrm{SNR} \gtrsim \sqrt{2 \ln \frac{N_{\text {pix }}}{\mathrm{SNR}}}=6.3,
$$

it is possible to comfortably identify $\mathrm{KBO}$ candidates without fear of massive contamination by noise spikes using a simple two-dimensional (2-D) search over the image. At this limit, there may be some contamination, but this could easily be vetted by examining successive images. There are two points to make about Equation (14). First, it assumes Gaussian statistics. This may not be valid for the case of a 2-D search. However, Equation (14) primarily serves as an entry point to the much larger (4-D, 5-D, and 6 -D) searches that I describe below, for which Gaussian statistics are valid. I therefore ignore this complication. Second, the SNR appears on both sides of the equation, meaning that the equation must be solved self-consistently. This poses no actual difficulties, since it appears inside a rather large logarithm factor on the rhs, but does call for an explicit remark.

Next, I consider 4-D searches over position (2-D, as above) and proper motion (2-D). I consider a search in a "proper motion" circle $\mu_{0}=12^{\prime \prime}$ day $^{-1}$ (relative to a $\mathrm{KBO}$ in circular motion at $r=40 \mathrm{AU}$. By conducting a 4-D search, I am implicitly assuming that the other two Cartesian phase-space coordinates $\left(\Pi\right.$ and $\left.\nu_{r}\right)$ are "not important". Explicitly, this assumption means that the parallax differences among the KBOs being searched lead to angular displacements of $<1$ pixel during the time of the search. For definiteness, I assume that KBOs of interest have $\Pi<\Pi_{0}=0.03$. The acceleration of Earth then leads to a differential pixel displacement of

$$
\Delta \theta_{\Pi}=\frac{1}{2}(\Omega t)^{2} \Pi_{0}=8 \theta_{\text {pix }}\left(\frac{\Delta t}{\text { day }}\right)^{2}
$$

due to parallax. Thus, a $4-\mathrm{D}$ search is restricted to $\Delta t<9 \mathrm{hr}$, and therefore requires a search radius of $\mu_{0} \Delta t$ and hence a total number

$$
N_{\mu}=\pi\left(\frac{\mu_{0} \Delta t}{\theta_{\text {pix }}}\right)^{2}=5300\left(\frac{\Delta t}{9 \mathrm{hr}}\right)^{2}
$$

of searches at each of $N_{\text {pix }}=3 \times 10^{9}$ pixels, for a total of $N_{\text {try }}=N_{\text {pix }} N_{\mu}=1.6 \times 10^{13}$ searches. In nine hours, there are approximately $N_{\text {im }}=30$ images. Hence this yields a SNR threshold of

$$
\mathrm{SNR} \gtrsim N_{\mathrm{im}}^{-1 / 2} \sqrt{2 \ln \frac{N_{\mathrm{try}}}{\mathrm{SNR}}-\ln N_{\mathrm{im}}}=1.38 .
$$

To dig to lower SNR, one must probe over longer durations, which requires 5 -D or 6 -D searches. To find the boundary, I adopt a radial-velocity search range $\Delta \nu_{r}=1 / 300$ corresponding to $\pm 2 \mathrm{~km} \mathrm{~s}^{-1}$ at $r=40 \mathrm{AU}$. Thus the radial velocity becomes important at $\Delta t \sim$ $\theta_{\text {pix }} /\left(\Delta v_{r} \Pi \Omega\right) \sim 9 \mathrm{hr}$, which is nearly identical to the onset of extra searches due to parallax. Combining all factors $\left((\Delta t)^{2}\right.$ for proper motions, $(\Delta t)^{2}$ for parallax, and $(\Delta t)^{1}$ for radial velocity), implies a search total of

$$
N_{\text {try }}=7 \times 10^{14}\left(\frac{\Delta t}{\text { day }}\right)^{5},
$$

implying a maximum search total for $\Delta t=72$ day of $N_{\text {try }}=1.4 \times 10^{24}$. Applying Equation (17), with $N_{\mathrm{im}}=5600$, yields a threshold $\mathrm{SNR} \gtrsim 0.15$, and so a theoretical limit of $H_{\text {vega }}=28.2$, or roughly $R \sim 29.6$. Recall from Equation (13) that even such extreme below-sky KBOs would have orbital parameter errors of order $1.5 \%$.

However, reaching this theoretical limit will be no picnic. One could convolve all the images with the PSF (e.g., Shao et al. 2014; Gould 1996), so that each search would require only $\sim 10 N_{\text {im }}$ floating point operations, and thus a total of $\sim 8 \times 10^{28}$ operations. This should be compared to the $\sim 10^{12}$ floating point operations per second (FLOPS) of a current graphics processing unit (GPU). One might imagine assigning $10^{4}$ GPUs to this task for a year, but this would only enable $3 \times 10^{23}$ operations, which is a factor $q \sim 2.5 \times 10^{5}$ short of what appears to be needed.

I will argue immediately below that this computational shortfall can probably be bridged by a combination of several factors. However, it is useful to ask how an arbitrary shortfall $q$ would impact the depth of the survey. From Equation (18) the number of trials scales $N_{\text {try }} \propto(\Delta t)^{5}$, while the number of computations for each trial is linear in $\Delta t$. Hence, a shortfall factor $q$ can be compensated by reducing $\Delta t$ by a factor $q^{1 / 6}=$ $8\left(q / 2.5 \times 10^{5}\right)^{1 / 6}$. Naively, this leads to an increase in the SNR limit by a factor $q^{1 / 12}=2.8\left(q / 2.5 \times 10^{5}\right)^{1 / 12}$. In fact, taking account of the impact on the logarithmic factor due to the smaller number of trials in Equation (17), the actual degradation is a factor 2.6, i.e., a limit $\mathrm{SNR}=0.38$, corresponding to $H<27.1$. Note that while the KBO is found using a restricted subset of the data, the full data set can still be used to estimate orbital parameters, so that the estimates of precision given in Section 5 remain valid.

For the survey to reach its maximum potential (i.e., 1 mag deeper than the above limit) requires either greater computing power or better search algorithms. Since the data will not be available for at least a decade, we should fold in a "Moore's Law" factor of 30 (assuming 
doubling time of 2 years), but this is still only $\sim 10^{25}$ operations. Since the number of operations scales $\propto$ $(\Delta t)^{6}$, this seems to permit analysis of data intervals $\Delta t \lesssim 16$ days, so only reaching SNR $\gtrsim 0.32$ and limiting magnitude $H_{\text {vega }}=27.4$.

In fact, it should be possible to go deeper using search techniques that are more clever than brute force trials. For example, one could begin by restricting the search to $\Delta t=16$ days as above, but initially cull out trajectories with $\Delta \chi^{2}>28$. This would capture exactly half of all ultimately recoverable KBOs (i.e., those with SNR $>0.15)$, since these would have $\left\langle\Delta \chi^{2}\right\rangle=28$, while at the same time suffering noise-spike contamination of "only" $\sim \exp (-28 / 2) / \sqrt{28} \sim 10^{-7}$. Now, of course, this would still result in $\sim 10^{14}$ noise spikes, but these could be vetted fairly efficiently, as follows. From Equation $(11), \sigma(\Pi)=4 \times 10^{-5}$ and $\sigma(\mu)=11$ mas day $^{-1}$. In fact, it is easily shown that the proper-motion error in the direction perpendicular to the ecliptic is smaller by $\sqrt{12 / 75}=0.4$. Therefore, even allowing for a $3 \sigma$ range for these two quantities, the total number of searches required for each such "preliminary candidate" is only $\sim 10^{7}$. If the procedure were repeated on 4 independent subsamples, it would recover $1-2^{-4} \sim 94 \%$ of all with $\mathrm{SNR}>0.15$. That is, almost full recovery with $\sim 8 \times 10^{25}$ FLOPS rather than $\sim 8 \times 10^{28}$ required for a brute-force search. That is, this algorithmic improvement, by itself, pushes down the magnitude limit by $\Delta H \sim(2.5 / 12) \log (1000) \sim 0.6 \mathrm{mag}$ relative to the brute force search.

One could imagine yet more clever ideas. It is premature to work these out in detail because the real algorithms would have to take account of not only operation speed but also memory access for processors that have not even been designed. The point is that it is not unrealistic to think that the theoretical limit of $\mathrm{SNR}>0.15$ can be reached, or at least approached within a few tens of percent. To reiterate what was said above, this limit corresponds to $H_{\text {vega }}<28.2$ or roughly $R<29.6$, with period errors $\sigma(P) / P \lesssim 1.5 \%$. And even if these improvements were not made, with present technology and a brute-force search one can still reach $H_{\text {vega }}<27.1$ or roughly $R<28.5$, with period errors $\sigma(P) / P \lesssim 0.6 \%$.

\subsection{Finding KBOs in Unexpected Orbits}

In the above treatment, the emphasis was on finding extremely faint KBOs, with SNR substantially below unity in individual exposures. To this end, it was necessary to restrict the search space to orbital parameters that are in some "expected" range, partly to limit the frequency of noise spikes, but mainly to make the search computationally tractable. However, it will also be possible to relax essentially all limits on orbital parameters down to $H \sim 24.1$, which is at the detection threshold $\mathrm{SNR} \sim 6.3$ required to distinguish a KBO from noise spikes in a single exposures. See Equation (14). Now, as emphasized above, Gaussian statistics do not apply to single images, but only to ensembles. However, the first point is that such non-Gaussian noise spikes could easily be vetted by comparing to neighboring images. Moreover, it would be straightforward to conduct searches over 1 day intervals, allowing for all parallaxes $\Pi<0.05$ and proper motions corresponding to all bound orbits. With about 90 exposures, such a search would reach SNR $\sim 1$ corresponding to $H \sim 26$, i.e., about 1 mag below the break. If this search found significant numbers of objects in unexpected (e.g., retrograde) orbits, then further searches could be fine-tuned to find fainter KBOs in similar orbits.

\section{Binary Companions and Mass MEASUREMENTS}

Regardless of the exact limit achievable for an ab initio search for KBOs, it is possible to reliably identify binary companions to all those that are found down to $H_{\text {vega }} \sim$ 29 , significantly fainter than the theoretical limit for isolated KBOs. Detection of such binary companions will lead to mass estimates and mass measurements of the parent KBO.

The reason that the search for companions can go deeper than the search for primaries is that the search space is smaller. The first task is therefore to quantify the size of the search space.

I parameterize the semi-major axis $a_{c}$, and hence the characteristic angular separation of the companion by

$$
\theta_{c} \equiv \frac{a_{c}}{a}=\eta\left(\frac{M}{M_{\odot}}\right)^{1 / 3}=\eta\left(\frac{\rho}{\rho_{\odot}}\right)^{1 / 3} \frac{D}{D_{\odot}} \simeq \eta \frac{D}{D_{\odot}}
$$

where $D$ and $\rho$ are the primary KBO effective diameter and density respectively, and where I have approximated $\left(\rho / \rho_{\odot}\right)^{1 / 3} \sim 1$. To be bound (within the Hill sphere), $\eta \lesssim 1$. This sets a strict upper limit on the separation. In fact, to remain bound over the lifetime of the solar system, the companion must be substantially closer, so this limit is conservative and is therefore robust against somewhat denser or less reflective KBOs. Hence, $\theta_{c} \sim \eta \times 1.4^{\prime \prime}(D / 10 \mathrm{~km})$ is constrained to be within a few arcsec near the magnitude limit ${ }^{3}$. The relative proper motion of the primary and companion are then of order

$$
\Delta \mu \sim \eta^{-3 / 2} \theta_{c} \Omega \Pi^{3 / 2} \sim \frac{7 \text { mas }}{72 \text { day }} \eta^{-1 / 2} \frac{D}{10 \mathrm{~km}}\left(\frac{a}{40 \mathrm{AU}}\right)^{-3 / 2}
$$

That is, near the detection limit (for primaries), one can search for companions simply by looking for nonmoving objects (i.e., those that move much less than 1 pixel relative to the primary during a 72-day campaign) within a few arcsec of the primary, i.e., $\sim 10^{3}$ trials for each primary. If we estimate that there will be $\sim 10^{4}$ KBO-primary detections, then SNR $\gtrsim 0.07$ is required to avoid noise-spike contamination. This implies a flux limit $H_{\text {vega }} \sim 29.0$, plausibly corresponding to a diameter $D \sim 7.5 \mathrm{~km}$.

\footnotetext{
${ }^{3}$ When converting from magnitudes to diameters, I adopt an albedo of 0.04 in R band and a "typical" distance of $40 \mathrm{AU}$, which yields $11 \mathrm{~km}$ at $R=29.6$ corresponding to $H_{\mathrm{vega}}=28.2$
} 
For such extremely faint KBO companions, one would measure only their position. However, if we consider more generally a pair of (for simplicity) equal brightness KBOs at a given SNR, their relative proper motion can be measured to a precision

$$
\sigma(\Delta \mu)=\sqrt{\frac{24}{N}} \frac{\sigma_{\text {ast }}}{\Delta t} \rightarrow \frac{35 \operatorname{mas~yr}^{-1}}{\mathrm{SNR}} .
$$

Then adopting, again for simplicity, $D=29 \mathrm{~km}(\mathrm{SNR})^{1 / 2}$ and $a=40 \mathrm{AU}$, we expect

$$
\Delta \mu \sim 100(\mathrm{SNR})^{1 / 2} \eta^{-1 / 2} \operatorname{mas~yr}^{-1} .
$$

This implies, very roughly, that such proper motions can be detected for $\eta \lesssim(\mathrm{SNR})^{3}$, i.e., for all bound companions at SNR $\gtrsim 1$ and for a rapidly declining fraction at fainter magnitudes.

Such proper motion estimates would, by themselves, give crude mass estimates for individual KBOs. But the ensemble of such measurements could be studied statistically to give the mean mass as a function of (solar system) absolute magnitude.

Much more detailed orbital motion could be obtained by shallow follow-up surveys. The ensemble of KBOs could be expected to disperse (relative to mean motion) at roughly $\sim 2 \mathrm{~km} \mathrm{~s}^{-1}$, corresponding to about $0.6^{\circ} \mathrm{yr}^{-1}$. Thus, one could use WFIRST itself (in its survey mode) to make brief (e.g., one day) surveys of the fields to which the KBOs were drifting one to several years after and/or before the discovery campaign in order to better characterize the orbits. The utility and characteristics of such observations could be much better assessed after logging the discoveries from the first campaign.

\subsection{Detection of Unresolved Companions}

Most KBO binaries detected to date have companions within $\sim 1 \mathrm{mag}$ of the primary, and this does not appear to be the result of selection, at least for the fairly bright $(R \lesssim 24)$ primaries in current samples (Noll et al. 2008). Many of these companions are also quite close, with a median separation near the WFIRST pixel size $p=$ 110 mas.

There are two methods of detecting such close companions that are of relatively comparably brightness: "orbiting" centroid of light and extended images.

There are three requirements to detect center of light motion: First, the actual separation must obey $\theta_{c} \lesssim p$. Otherwise, given the undersampled PSF, the companion would be directly detectable. Second, the companion must complete a large fraction of an orbit. Otherwise, the centroid of light motion will simply be absorbed into the KBOs orbital parameters with respect to the Sun. To be moderately conservative, and for simplicity, I interpret this requirement as completing one orbit during $\Delta t=72$ days. Third, the signal must be sufficiently high to distinguish centroid motion from noise spikes.

Figure 1 shows that the fractional displacement of the centers of light and mass (assuming same density and albedo) peaks broadly $7 \% \lesssim f_{\mathrm{cl}} \lesssim 9 \%$ for

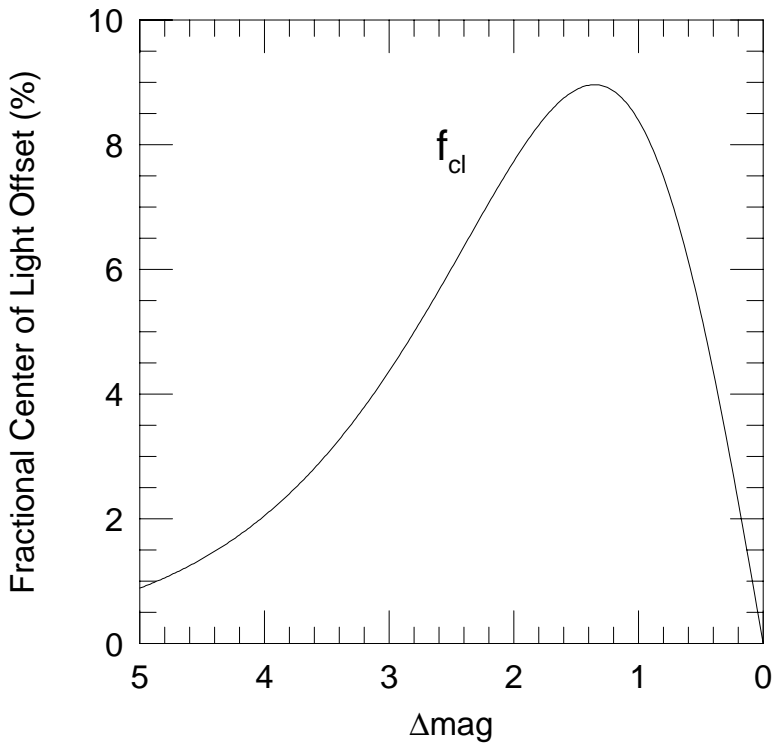

Figure 1. Offset of binary center of light from center of mass, as a fraction of physical separation under the assumptions that the two components have equal density and equal albedo. There is a broad peak $7 \%<f_{\mathrm{cl}}<9 \%$ for $0.72<\Delta$ mag $<2.2$.

$0.72<\Delta$ mag $<2.2$. Assuming that the rms projected amplitude is $\sqrt{2}$ smaller than the physical separation, and that a $7 \sigma$ signal is required for detection, this implies a minimum SNR for each of the 5600 measurements of

$$
\mathrm{SNR} \gtrsim 7 \sqrt{\frac{2}{5600}} \frac{p}{f_{\mathrm{cl}} \theta_{c}} \gtrsim 1.65 \frac{p}{\theta_{c}}\left(\frac{f_{\mathrm{cl}}}{0.08}\right)^{-1},
$$

which corresponds to $H<25.5$ (or $R \lesssim 26.9$ ).

From the definition of $\eta$, the boundary $P_{c}=$ $\Delta t=72$ days implies $\eta=(a / \mathrm{AU})^{-1}\left(P_{c} / \mathrm{yr}\right)^{2 / 3}=$ $0.0085(a / 40 \mathrm{AU})^{-1}$. Hence, imposing $\theta_{c}=p$ in Equation (19) at this period yields $D=p D_{\odot} / \eta=$ $88 \mathrm{~km}(a / 40 \mathrm{AU}$ ), which corresponds to $H \sim 23.7$ (or $R \sim 25.1)$ at $a \sim 40 \mathrm{AU}$.

These two calculations show that there is considerable parameter space for detection of this effect. In Figure 2, I show the SNR for light centroid motion as a function of binary separation $\theta_{c}$ for a range of KBO brightnesses from $H=23$ to $H=25$. The curves are displayed only for periods $P<\Delta t=72$ days and so "cut off" at the right for the fainter KBO tracks. Note in particular that at $H=23$ (roughly $R=24.4$, so almost completely encompassing the region of present studies), the separation threshold is $\theta_{c}=11$ mas,

It is important to remark that this method is critically dependent on excellent overall WFIRST astrometry. For example, the $\sim 1$ mas "light centroid orbits" for $H=23 \mathrm{KBOs}$ at the detection limit in Figure 2 would not be detectable without the excellent world coordinate system described in Section 4.

For truly equal-mass (and equally reflecting) KBOs, the center of mass and center of light will be identical, 


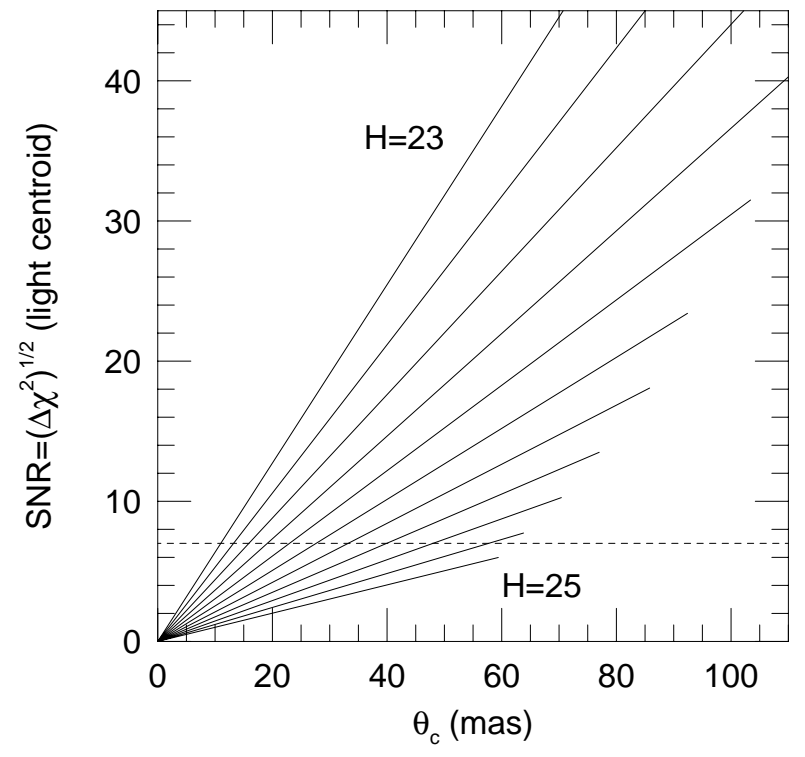

Figure 2. Signal-to-noise ratio $\left[\left(\Delta \chi^{2}\right)^{1 / 2}\right]$ for orbiting KBOs at a range of separations that are less than the WFIRST pixel size $\theta_{c}<p=110$ mas. The KBO brightness ranges from $H_{\text {vega }}=23$ to $H_{\text {vega }}=25$ as indicated. Tracks end to the right when the orbital period $P=\Delta t=72$ days, the duration of an observing campaign. Longer period orbits would have substantially lower signal. The calculations assume $f_{\mathrm{cl}}=8 \%$ (see Figure 1 ) and make a variety of assumptions listed in the text, including $D\left(H_{\text {vega }}=24.6\right)=58 \mathrm{~km}$, $a=40 \mathrm{AU}$, and rms projected separation equal to $\theta_{c} / \sqrt{2}$.

implying identically zero offset between mass and light. For small differences, $f_{\mathrm{cl}}=0.11 \Delta$ mag (see Figure 1). However, this near equal-mass regime is the most sensitive to image elongation. A detailed study of the limits of detectability is beyond the scope of the present work. However, I would emphasize that the combined images for the KBOs in the magnitude range shown in Figure 2 will be fairly deep. For example, at $H_{\text {vega }}=25$, the combined image will have $\mathrm{SNR}=200$ and will be resolved at subpixel scales because it is composed of 5600 dithered images.

\section{KBO SIZE ESTIMATES FROM OCCULTATIONS}

One of the major advantages of KBO searches in dense microlensing fields is the large number of occultations that are automatically observed. These occultations can yield statistical information on the relation between effective diameter and reflected light, i.e., the albedo. In principle, these occultations might give rise to astrometric noise, but I show that this effect is negligible.

\subsection{Occultation Measurements}

As I will show below, in contrast to the general astrometric measurements, which are below sky $\left(H_{\text {vega }}<\right.$ 21.7), the occultations will be of sources that are above sky. In particular, these will be substantially brighter than almost all the KBOs. Thus, the photon noise will be dominated by the occulted star. In addition, there is noise from unmodeled variability of the KBO. However, here I will assume that the variability is well-modeled (at least to the level of the photon noise for individual measurements) so that only photon noise need be considered.

However, there is a third form of uncertainty in interpretation of occultations: the flux of the occulted star. That is, what we would like to know is the fraction of the exposure that the star was occulted, which is just equal to the missing flux divided by the occultedstar flux. Hence, for an occultation to be usefully interpreted, even statistically, the occulted star must be identified reasonably securely. The average density of stars down to $H_{\text {vega }}<(21,22)$ is $(0.03,0.05) p^{-2}$ (i.e., "per pixel"). I assume that sources can be reasonably photometered down to $H_{\mathrm{vega}, *}=21$, keeping in mind that the "exact" location of the source (to a small fraction of a pixel) is known from the occultation itself together with the known KBO orbit. This precise location of the occulted star (together with subpixel resolution from 40,000 images) makes it much easier to disentangle the occulted star from significant blends.

The transverse velocity of the KBO in the Earth frame will generally be dominated by reflex motion of Earth, which will have an rms value of $v \sim 10 \mathrm{kms}^{-1}$ during the 72 days of observations near quadrature. I will adopt this as a typical value in my initial, simplified treatment.

I begin by examining the case of KBOs with $H=24.6$ (0.5 mag brighter than the break), which have diameters $D \sim 58 \mathrm{~km}$, and hence a maximum self-crossing time of $D / v \sim 5.8 \mathrm{~s}$, i.e., $11 \%$ of an exposure time. Thus, assuming that the occultation is entirely contained in the exposure, this implies a drop of only $11 \%$ of the occulted-star flux. At $H_{*}=21$, the (above-sky) photometric error is $\sim 1.4 \%$, implying that such a flux deficit would be detected at the $11 / 1.4 \sim 8 \sigma$ level. This immediately raises the question of what is the threshold at which we should say that an occultation has been "detected" in the face of (negative) noise spikes? To evaluate this, we must first estimate the effective number of "trials". I conservatively estimate that $H<21$ star positions can be measured (independent of the occultation) to 0.3 pixels. Since the position of the KBO is known to much higher precision, the probability that a KBO will land near enough such a star to be consistent with an occultation is $0.03 \times \pi(0.3)^{2}<1 \%$ We must consider all 6900 observations (including $Y$-band and, of course, those landing near bright stars). Hence, there will be a total of $\sim 70$ "trials" per KBO. As I will show shortly, we expect only about one real occultation from these 70 trials. This means that a $3 \sigma$ cut would yield $\mathrm{a} \sim 20 \%$ false positive rate. This would be acceptable if one carried out a very careful statistical study but to be conservative, I adopt a $4 \sigma$ threshold, for which the (false positive)/(real occultation) rate is about $0.6 \%$.

Ignoring this threshold for the moment, the rate of 
occultations per $\mathrm{KBO}$ is

$$
\begin{aligned}
N_{\mathrm{oc}}= & \frac{N_{\mathrm{obs}} n_{21} D v t_{\text {exp }}}{a^{2}} \\
= & 0.6 \frac{N_{\mathrm{obs}}}{6900} \frac{n_{21}}{0.03 p^{-2}} \frac{D}{58 \mathrm{~km}} \frac{v}{10 \mathrm{~km} \mathrm{~s}^{-1}} \\
& \times \frac{t_{\exp }}{52 \mathrm{~s}}\left(\frac{a}{40 \mathrm{AU}}\right)^{-2},
\end{aligned}
$$

where $n_{21}$ is the surface density of $H_{*}<21$ stars. In the example that we are considering, the maximum dip is $8 \sigma$, while the adopted threshold $4 \sigma$. This means that exposures that begin more than half-way through an occultation will fall below the threshold, but these will be exactly compensated by the ones that end during the occultation but less than half way through it. More generally, such edge effects will not exactly cancel and must be taken into account. However, for the present case, Equation (24) does not require adjustment. In addition, KBOs that transit chords that are less than half the diameter will fall below the detection threshold. This corresponds to $1-\sqrt{1-(4 / 8)^{2}} \sim 14 \%$ in the present case (approximating the projected form as a circular disk), but will vary for other parameters.

In particular, this means that at $H=24.6$, there are about 0.5 occultations per KBO. As I discuss in Section 12, this implies that among the several thousand KBOs discovered, there will be over one thousand occultations.

Because the occultation time is short compared to the exposure time, the flux decrement (combined with occulted-star flux estimate, and the known instantaneous KBO proper motion and distance) directly yields the chord length. The frequency of such occultations gives one an estimate of the mean diameter (at fixed absolute magnitude) while the peak of the chord-length distribution gives another. Formally, these are respectively transverse and parallel to the direction of motion, but these are expected to be statistically identical. If the typical detections are $8 \sigma$, then the second measure will have 8 times smaller formal error than the first, but the existence of two independent measurements will provide a useful check on the systematics.

The KBO break at $H=25.1$ has the peak occultation rate. At fainter magnitudes, the KBO luminosity function is flat (and later, perhaps, falling), while the number of occultations falls as $D$, i.e., as $10^{-0.2 H}$. Moreover, at about 1 mag fainter than the break, the flux decrement drops below the detection threshold (for fiducial parameters), so one is restricted to brighter (hence rarer) occulted stars and/or KBOs observed at times that they are moving more slowly relative to Earth than average. On the other hand, KBOs that are 1 mag brighter than the break are 4 times less common (Bernstein et al. 2004). This is compensated by the factor $~ 1.6$ larger diameter entering Equation (24), but still leads to a factor 2.5 (i.e., inversely proportional to flux) fall in the total number of occultations.

I predict the number of occultations as a function of KBO magnitude using a more detailed calculation in
Section 12.

\subsection{Occultation Noise}

The first point to note is that occulted stars are aligned with the geometric center of the KBO much more closely than the astrometric precision of individual measurements. Consider, for example, a KBO at $40 \mathrm{AU}$ and $H \sim 24.6$ (0.5 mag brighter than the break in the luminosity function) with a diameter of $D \sim 60 \mathrm{~km}$. To be occulted, the star must be aligned to within $D / 2 a \sim 1$ mas, whereas the astrometric precision of the measurement is only $\sim 25$ mas. Hence, the only impact on measurement is the loss of net flux from the KBO due to a "hole" caused by the lost light of the background star. Moreover, it is only the absolute value of the flux change at the KBO position that enters the astrometric precision. That is, it is just as easy to centroid a "hole" as a "bump", provided it is recognized that there is hole (i.e., that an occultation is taking place). Hence, it is really only occultations within about a half magnitude of the KBO brightness that have an adverse impact on astrometry.

To evaluate the practical impact, let us consider two cases, one at $\sim 1 \mathrm{mag}$ above the break with $H \sim 24$ $(D \sim 75 \mathrm{~km})$ and the other near the limit $H \sim 28(D \sim$ $12 \mathrm{~km})$. Since the KBOs are observed near quadrature, I adopt transverse relative velocities of $v=10 \mathrm{~km} \mathrm{~s}^{-1}$, which implies that the KBOs near the break have a self-crossing time of about 7 seconds, i.e., $\sim 1 / 7$ of the exposure times, $t_{\exp }=52 \mathrm{~s}$. Hence, for an occultation to reduce the flux by an amount similar to the KBO brightness requires that the star has $H_{*} \sim 22$. The number of stars within a half magnitude of this value is similar to the total number brighter than it, which led (above) to an estimate of less than one occultation per KBO.

If we now consider KBOs near the limit (i.e., 40 times fainter), the duration of occultation is $40^{1 / 2} \sim 6.5$ times shorter, implying that the "problematic" stars are a factor 6.5 (i.e., $2 \mathrm{mag}$ ) fainter, and so only slightly more numerous. On the other hand, the cross section is 6.5 times smaller, so the net effect is even smaller.

\section{EFFECTS OF GEOSYNCHRONOUS ORBIT}

If WFIRST is in geosynchronous orbit, this will have almost no effect on the calculations in this paper. However, it will have some modest benefits for the followup observations proposed in the previous section.

Geosynchronous orbit induces diurnal parallax of amplitude $\Pi_{\text {geo }}=\epsilon \Pi$ where $\epsilon \sim 1 / 4000$. Since the target fields lie near the plane of this motion, diurnal parallax enables a fractional distance measurement

$$
\sigma(\Pi)=\sqrt{\frac{2}{N}} \frac{\sigma_{\text {ast }}}{\epsilon}
$$

where $N$ is the number of observations in some time that is at least one day. Equating this to the second expression in Equation (11), one finds that orbital parallax overtakes geosynchronous parallax at $(\Delta t)_{\Pi}=$ 


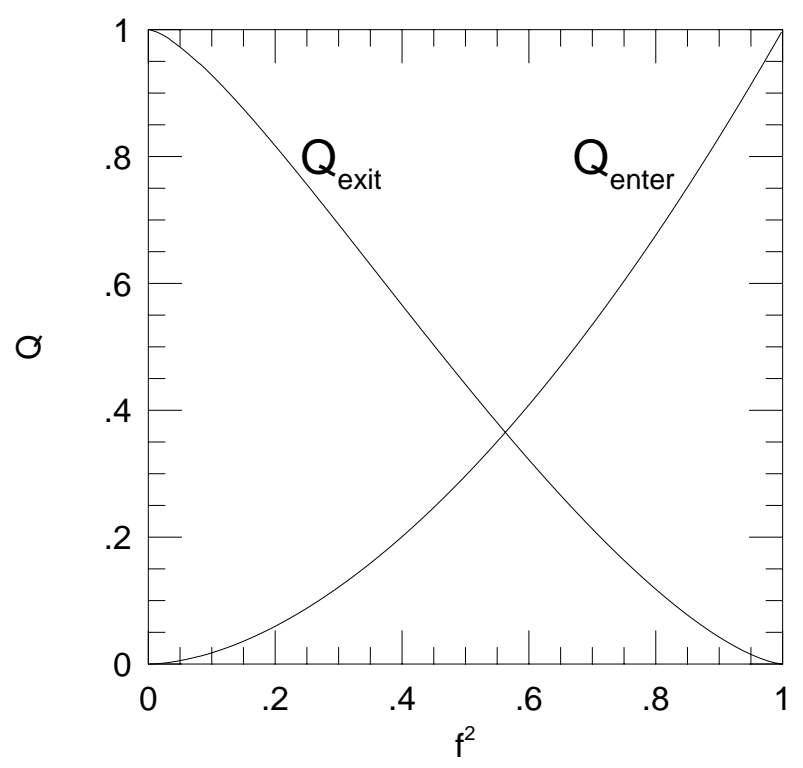

Figure 3. Degradation factor $Q$ for the error in the radial velocity measurement (by far the worst measured phase-space coordinate) as a function of $f$, the fraction of a campaign that is spent outside (inside) the microlensing field by a KBO that exits (enters) during the campaign. For low $f$, $Q_{\text {exit }}$ is modest for those exiting, and this is compensated at high $f$ by those entering. Only about $40 \%$ of all KBOs that are initially in the field exit during a campaign, so the net effect of exits/entrances is modest. Abscissa is $f^{2}$ because the distribution of field area is uniform in this quantity.

$360^{1 / 4} \epsilon^{1 / 2} \Omega^{-1} \sim 4.0$ day. A similar calculation for the radial velocity determination (now assuming at least several days of data) yields a crossover that is just slightly larger: $(\Delta t)_{v_{r}}=(35 / 27)^{1 / 4}(\Delta t)_{\Pi} \sim 4.3$ days. Thus, diurnal parallaxes are really only useful for the handful of KBOs that briefly cross the field, or for followup observations that are carried out over of order one day in non-campaign years.

Similarly, the diurnal parallaxes do not significantly complicate the search. Diurnal motion is $\sim \epsilon \Pi \sim 1.3^{\prime \prime}$. This implies an absolute minimum of about 20 pixels in the parallax "direction" of the search space, contrary to the assumption of the "position and proper motion" search described at the beginning of Section 6. However, this simplified search was actually presented only for didactic purposes. The real searches described further along in that section already have a much larger parallax footprint. Hence, diurnal parallax due to geosynchronous orbit has essentially no impact either on the precision of measurement or the difficulty of searching for KBOs.

\section{EDGE EFFECTS}

Up to this point, I have assumed that the KBOs remain in the field for the entire 72 day campaign. However, KBOs that are initially near the ecliptic East (West) edge of the field for a Spring (Autumn) campaign will move off the field as Earth approaches the equinox and will return into the field later on, whereas other KBOs that initially lie just beyond the ecliptic West (East) edge will enter the field and then leave it. Now, as shown in Section 5, the majority of KBOs do in fact remain in the field for the full 72-day campaign, and so to zeroth order one could in principle just ignore these edge effects. However, here I show that these effects actually play only a small role even at first order.

Since the apparent motion of the KBOs is dominated by reflex motion of Earth, I calculate the time spent within the field as though this were the only cause. Then the time spent inside (and outside) the chip is symmetric about the midpoint of the campaign. I first consider the KBOs that begin within the field and parameterize the time spent out of the field by $f$, i.e., they spend a time $f \Delta t$ outside the field. I focus on the precision of the radial velocity measurement because it is by far the weakest of the six phase space coordinates. Repeating the integral that led to Equation (10) but excluding observations during the time spent outside the field, $f \Delta t$, yields

$$
\begin{aligned}
Q_{\text {exit }}^{2}(f) & \equiv \frac{c_{33}(\text { full })}{c_{33}(\text { partial })} \\
& =\frac{1-(25 / 4) f^{3}\left(1-1.68 f^{2}+f^{4}\right)+f^{10}}{1-f^{3}} .
\end{aligned}
$$

This function declines monotonically with $f$, but there is a compensating effect of KBOs entering the other side of the field and spending time $f \Delta t$ within the field. Since these entering KBOs are observed continuously, the corresponding calculation is trivial,

$$
Q_{\text {enter }}(f)=f^{3.5}
$$

Finally, I note that one must account for the fact that the distribution of KBOs leaving (or entering) the field for a time $f \Delta t$ is not uniform in $f$ but rather in distance from the edge of the field at the midpoint of the campaign, which scales $\propto f^{2}$. That is, $75 \%$ of all KBOs that leave (or enter) the field do so for more than half the campaign.

To visualize these effects, I plot $Q_{\text {exit }}$ and $Q_{\text {enter }}$ versus $f^{2}$ in Figure 3. When taking account of both effects, the maximum degradation factor is $Q \sim 0.365$ at $f^{2} \sim 0.57$. This factor is modest given the huge range of KBO brightness being probed. Moreover, it is compensated by the fact that twice as many KBOs are probed at these distances from the edge of the field. In brief, the total area of the fields in all six campaigns, $6 \times 2.8 \mathrm{deg}^{2} \sim 17 \mathrm{deg}^{2}$ is a reasonable estimate of the effective area of the KBO survey.

\section{KBO LOST AND FOUND}

Much of the science that can be extracted WFIRST KBOs will be derived directly from the WFIRST data themselves. This includes the distribution of KBOs as a function of orbital parameters, colors, size, binarity, etc. However, there are a number of applications that 
could require re-observing a detected KBO one or many years later. For example, as mentioned in Section 7, one might want to obtain late-time observations of a binary companion in order to better estimate the corresponding primary's mass. As another example, one might want to measure a late-time position of a KBO that has a moderate-precision period in order to precisely determine whether it was actually on, or simply near a resonance.

Here I show that all the KBOs discussed above can be unambiguously recovered even 10 years after they are discovered in WFIRST data. For illustration, I focus on those at the detection limit, for which I have estimated period errors of $\sigma(P) / P \sim 1.5 \%$. I will then briefly remark on the situation for brighter KBOs.

After 10 years, a KBO at $a \sim 40 \mathrm{AU}$ with $1.5 \%$ period error will lie somewhere along a "well-defined arc", with a $1 \sigma$ position error along that arc of $\sigma(\theta) \sim$ $1.5 \%\left(10 / 40^{3 / 2}\right) \times 360^{\circ}=13^{\prime}$. Considering that a conservative search might look within $\pm 2.5 \sigma$, this implies a search along an arc of $\sim 1^{\circ}$. On the other hand, as discussed below Equation (11), the deviations orthogonal to this track are at least 300 times smaller than along it, implying a search width $\lesssim 12^{\prime \prime}$. Given that the surface density of KBOs at this limit is only $\sim 100 \mathrm{deg}^{-2} \mathrm{mag}^{-1}$, there is only a small probability that there will be even one other KBO of similar magnitude in the search zone. Because these KBOs are well below sky, re-detection will necessarily require several epochs and therefore will automatically return a proper-motion measurement that will almost certainly distinguish between the (possible) candidates in this zone.

Brighter KBOs will, of course, be much easier to find. For example, at the break in the KBO luminosity function $(H \sim 25.1)$, the period errors will be smaller by a factor 17 , so that the search zone will be smaller by the same factor in each dimension, i.e., $3.5^{\prime} \times 0.7^{\prime \prime}$.

\section{Expectations Based on Previous KBO SURVEYS}

\subsection{Expected KBO Detections}

The WFIRST microlensing survey will, without any modification, probe KBOs down to $H_{\text {vega }} \sim 28.2$ over an area of $\sim 17 \mathrm{deg}^{2}$ and yield orbits with period precision of $1.5 \%$ at the magnitude limit (and much better at brighter magnitudes). For my adopted conversion $R-H=1.4$, this corresponds to $R \sim 29.6$. How does this compare to previous deep surveys?

Bernstein et al. (2004) searched $0.019 \mathrm{deg}^{2}$ down to $R \leq 29$ (strictly, $m_{606} \leq 29.2$ ) using $22 \mathrm{ks}$ exposures with ACS on the HST. They found three new objects. For the two of these with $m_{606}>28$, they obtained only crude $(\sim 30 \%)$ orbital parameters. They found no KBOs $m_{606}>28.3$ even though they had near- $100 \%$ completeness to $m_{606}=29$, implying that the distribution is flat (or falling) beyond $R=28.5$. Combining their results with previous work at brighter magnitudes,

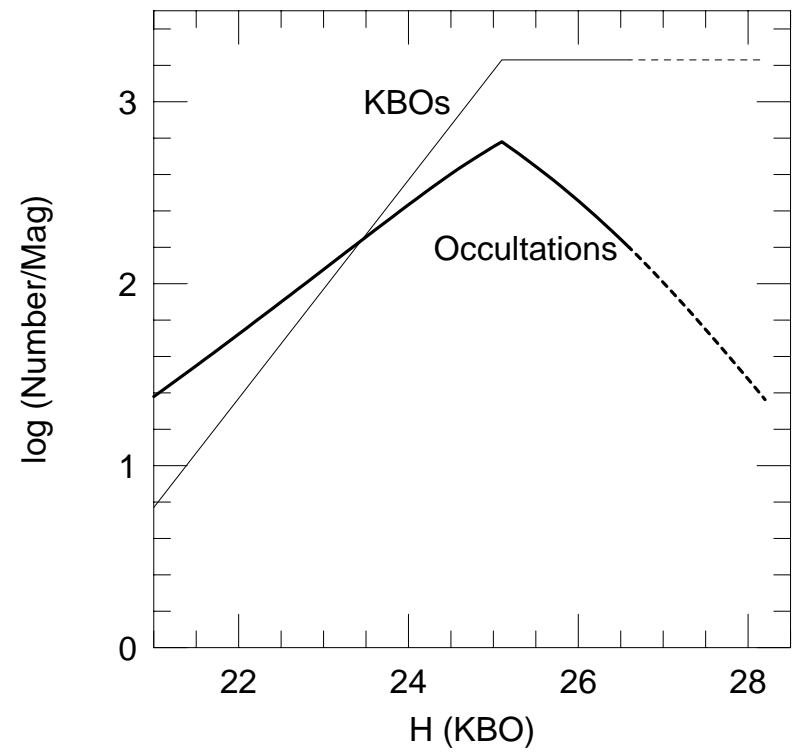

Figure 4. Predicted number of KBO detections (solid) and $\mathrm{KBO}$ occultations (bold) per magnitude that can be extracted from the WFIRST microlensing survey. The detections are derived by multiplying the Bernstein et al. (2004) KBO luminosity function by $17 \mathrm{deg}^{2}$. The curve is dashed for $H>27.1$ because the KBO frequency is not presently known in this regime. The calculation of the occultation rate per $\mathrm{KBO}$ is outlined in Sections 8.1 and 12.2. In particular, I count only occultations of $H_{*}<21$ stars that can be detected with at least $4 \sigma$ significance.

they fit a relation that can be expressed as,

$$
\frac{d^{2} N}{d R d \Omega}=\frac{100}{\operatorname{mag} \operatorname{deg}^{2}} 10^{0.6(R-26.5)} \quad(R \leq 26.5)
$$

and then a roughly flat distribution $\sim 100 \mathrm{mag}^{-1} \mathrm{deg}^{-2}$ for $26.5<R<28.5$ (and possibly beyond). The rising part of this distribution contains $\sim 70 \mathrm{deg}^{-2}$. Based on this estimate, we can expect that WFIRST would detect 4500-6500 KBOs. The Bernstein et al. (2004) relation (Equation (28)) scaled by $17 \mathrm{deg}^{2}$ is shown in Figure 4. The dashed part of the curve indicates an extension into the unmeasured regime.

\subsection{Expected Occultations}

In Section 8, I presented a simplified calculation of the KBO occultation rate, in order to illustrate the basic physics. In Figure 4, I show the results of a more detailed calculation including all the elements outlined in Section 8. In particular, I integrate over the full range of Earth-KBO relative velocities, which is mainly driven by the changing reflex motion of Earth but also includes a small component due to intrinsic KBO motion. I continue to demand $4 \sigma$ detections and consider only occultations of sources $H \leq 21$. The principal results from the simplified calculations are all confirmed.

One point to further note is that at the bright end of the distribution shown in Figure $4(H=21)$ there are 
about four occultations per KBO. Although this applies to only a half dozen objects per magnitude, it does hold out the hope that some shape information can be extracted, particularly if high-resolution followup imaging can determine the precise source location and therefore the precise impact parameter of the occultation.

\subsection{Expected Binary KBO Detections}

Statistics on KBO companions are available primarily for relatively bright primaries $R \lesssim 24$, corresponding to $H \lesssim 22.6$. These have a median separation of $\sim 100$ mas, and are primarily of near-equal brightness, with a substantial majority roughly uniformly distributed over $1>\Delta$ mag $>0$. About $22 \%$ of classical and $5 \%$ of other KBOs have such companions (Noll et al. 2008). Essentially all analogs of these companions will be found by WFIRST for the bright KBOs in its field. If there are similar companions down to $H=24$, then the total number of such binaries will be about 200. As shown by Figure 2, at fainter magnitudes the close binaries become less accessible and then inaccessible for $H>25$. Moreover, there is essentially no information on the frequency of companions at these magnitudes. Hence, while the unexplored parameter space is fairly large, there is no reliable way to estimate the number of companions in these regimes.

\section{Application to Ground-Based Microlensing Surveys}

There are several ground-based microlensing surveys that could in principle be searched for KBOs, including the ongoing OGLE-IV and MOA-II surveys, as well as the KMTNet survey, which is about to begin. Here I describe some relatively low-effort "entry points" into these data sets and briefly sketch extensions that would probe much deeper. The "entry point" searches could be carried out using a single night of data and would yield of order a dozen KBOs. The extensions could reach within $\sim 2$ mag of the break in the luminosity function $R \sim 26.5$, thus multiplying this number several fold.

The calculations below are based on the summaries provided by Henderson et al. (2014) of the telescope, detector, and observing characteristics of the OGLE and KMTNet surveys. In particular I adopt a photoelectron rate $\dot{\gamma}=4.91(D / 1.6 \mathrm{~m})^{2} \mathrm{~s}^{-1}$ at $I=22$, where $D$ is the diameter of mirror, an ambient background of $I=18.8 \mathrm{mag} \operatorname{arcsec}^{-2}$, and an effective PSF area of $\Omega_{\text {back }}=1.7 \times 4 \pi(\mathrm{FWHM})^{2} / \ln 256$. I also assume that the below-sky errors are 1.3 times larger than the photon noise. As noted by Henderson et al. (2014), the $I=18.8$ background, based on current OGLE-IV data, is not understood, and might be improved by further technical developments. Hence, these numbers are conservative.

OGLE observes three fields, totaling about $5 \mathrm{deg}^{2}$, with a cadence of $3 \mathrm{hr}^{-1}$, with exposures of about 100 $\mathrm{s}$, using a $1.3 \mathrm{~m}$ telescope. The fields can be observed for 10 hours per night (so 30 observations) for about one month centered on the summer solstice. I assume that one of these nights is clear, with low moonlight, and very good seeing of FWHM $\sim 1^{\prime \prime}$, and that $90 \%$ of the observations are not seriously affected by above-sky stars, cosmic rays, or pixel defects. Each (below-sky) observation then has $\mathrm{SNR}=10^{0.4(22.5-I)}$. The observations take place at opposition rather than quadrature (as for WFIRST), which means one must consider a proper motion rectangle of $\left(30 \mathrm{~km} \mathrm{~s}^{-1} / 30 \mathrm{AU}\right) \times$ $\left(3 \mathrm{~km} \mathrm{~s}^{-1} / 30 \mathrm{AU}\right)$, which for 10 hours of observations and $1^{\prime \prime}$ seeing implies 300 searches per resolution element or a total of $2 \times 10^{11}$ searches. This requires a total $\mathrm{SNR}=7$, which in $30 \times 0.9=27$ observations can be achieved for $I=22.2 \mathrm{KBOs}$, corresponding to $R=22.7$. There are roughly 0.4 such KBOs per square degree, implying that a few would be expected in the $5 \mathrm{deg}^{2}$ high-cadence OGLE field. However, this could be repeated for each of 5 years of OGLE-IV archival data, yielding about 10 KBOs.

KMTNet will have three $1.6 \mathrm{~m}$ telescopes, two of which will be at excellent and somewhat overlapping sites in Chile and South Africa. Its cadence at each will be $6 \mathrm{hr}^{-1}$, with $120 \mathrm{~s}$ exposures over $16 \mathrm{deg}^{2}$. Combining these and adopting a slightly worse mean seeing of $1.2^{\prime \prime}$ (to account for the greater difficulty of coordinating observations from two sites), yields a similar $\mathrm{SNR}=10^{0.4(22.6-I)}$. Then, taking account of the four times greater number of exposures, the limit of detectability is $I \sim 23.0$ corresponding to $R=23.5$. This would yield roughly 1 KBOs per square degree or a total of about 15 .

Once identified, these KBOs would yield excellent orbits because microlensing fields cover about $100 \mathrm{deg}^{2}$ over many years, albeit at lower cadence.

Over a whole season, KMTNet would observe any given KBO roughly 10,000 times from Chile and South Africa, of which $1 / 4$ would be in good seeing, good transparency, and with low Moon background. As with WFIRST, the search space would be much larger than for a simple one-night search, so I adopt a similar $\Delta \chi^{2}=115$ threshold. This leads to a detectability limit of $I=24.3$, or $R \sim 24.8$, i.e., about 100 KBOs. Note that in contrast to WFIRST detections, one need not be extremely rigorous about eliminating noise spikes at the detection phase because these will be automatically vetted when the KBO is tracked to additional seasons to measure its orbit.

\section{Conclusions}

Space-based microlensing surveys are an extremely powerful probe of KBOs basically because microlensing motivates very high cadence observations over long time baselines and fairly wide fields that happen by chance to lie near the ecliptic. The very large number of images allows one to construct essentially noiseless (compared to the individual images) templates, and so construct essentially blank images from the "crowded" fields via image subtraction.

In particular, the WFIRST microlensing survey, 
without any modification, can yield 4500-6500 KBOs down to $H_{\text {vega }}=28.2$. The last magnitude of such a search requires algorithmic and/or hardware development to carry out the computations in a timely manner. However, the more restricted search to $H_{\text {vega }}=27.1$ (with 4500-5000 KBOs) can be carried out by simple brute force searches using today's technology.

Because the detections arise from a near-continuous time series over much less than a year, and centered at quadrature, the statistical characterization of the orbital parameters is best understood in a 6 -D cartesian framework. The same framework allows one to directly map the expected (or, more generally, allowed) orbit space into a cartesian search space. In particular, I find that for WFIRST, the period errors scale as $\sigma(P) / P \sim$ $0.09 \% \times 10^{0.4\left(H_{\text {vega }}-H_{\text {break }}\right)}$, where $H_{\text {break }}=25.1$ is the break in the luminosity function.

Binary companions that are separated by a few pixels can be found down to about $H_{\text {vega }} \sim 29$, regardless of the limit of the primary search. The limit is deeper because the search space is smaller, implying fewer noise spikes. These binaries can provide statistical mass information, or if followed up by additional observations, individual mass measurements.

Binary companions with separations down to 0.1 pixels (11 mas) can be found for roughly equal (but not exactly equal) masses for primaries $H \leq 23$ from the offset between centers of mass and light, and for larger subpixel separations down to $H \sim 25$. Exactly (or very nearly) equal-mass binaries at sub-pixel separations can be detected from image elongation. Analogs to essentially all binaries currently being found $(R \lesssim 24$, $\left.\theta_{c} \gtrsim 0.01^{\prime \prime}\right)$ will be found by WFIRST, but it will also probe a huge parameter space of binary companions that has not yet been explored.

A side benefit of the fact that microlensing searches are carried out in the most crowded fields (prior to image subtraction) is the high probability of occultations. On average, each KBO at the break will occult 0.4 stars with $H_{*}<21$ (so reliably detected in the deep drizzled image) and with at least $4 \sigma$ detections. Over 1000 occultations of detected KBOs will enable measurement of the KBO albedo as functions of orbital properties and absolute magnitude.

Finally, using the same techniques outlined in this paper, it should be possible to find roughly $100 \mathrm{KBOs}$ using current and soon-to-be-initiated ground-based microlensing surveys.

\section{ACKNOWLEDGMENTS}

I thank Matthew Penny, Scott Gaudi, and Radek Poleski for helpful suggestions. This work was supported by NSF grant AST 1103471 and NASA grant NNX12AB99G.

\section{REFERENCES}

Alard, C., \& Lupton, R. H. 1998, A Method for Optimal Image Subtraction, ApJ, 503, 325

Bernstein, G. M., Trilling, D. E., \& Allen, R. L. 2004, The Size Distribution of Trans-Neptunian Bodies, AJ, 128, 1364

Doressoundiram, A., Boehnhardt, H., Tegler, S. C., \& Trujillo, C. 2008, Color Properties and Trends of the Transneptunian Objects, in The Solar System Beyond Neptune, (eds.) M. A. Barucci, H. Boehnhardt, D. P. Cruikshank, and A. Morbidelli (Tucson: University of Arizona Press), 91

Gomes, R., Levison, H. F., Tsiganis, K., \& Morbidelli, A. 2005, Origin of the Cataclysmic Late Heavy Bombardment Period of the Terrestrial Planets, Nature, 435, 466

Gould, A. 1996, Theory of Pixel Lensing, ApJ, 470, 201

Gould, A. 2003, $\chi^{2}$ and Linear Fits, arXiv:astro-ph/0310577

Gould, A., \& Yee, J. C. 2013, Microlens Surveys are a Powerful Probe of Asteroids, ApJ, 767, 42

Henderson, C. B., Gaudi, B. S., Han, C., et al. 2014, Optimal Survey Strategies and Predicted Planet Yields for the Korean Microlensing Telescope Network, ApJ, 794, 52

Monet, D. G., Levine, S. E., Canzian, B., et al. 2003, The USNO-B Catalog, AJ, 125, 984

Noll, K. S., Grundy, W. M., Chiang, E. I., et al. 2008, Binaries in the Kuiper Belt, in The Solar System Beyond Neptune, (eds.) M. A. Barucci, H. Boehnhardt, D. P. Cruikshank, and A. Morbidelli (Tucson: University of Arizona Press), 345

Slipher, V. M. 1930, The Discovery of a Solar System Body Apparently Trans-Neptunian, Lowell Observatory Observation Circular (March 13)

Slipher, V. M. 1930b, A Trans-Neptunian Planet, Popular Astronomy, 38, 187

Shao, M., Nemati, B., Zhai, C., et al. 2014, Finding Very Small Near-Earth Asteroids Using Synthetic Tracking, ApJ, 782, 1

Sheppard, S. S., Udalski, A., Trujillo, C., et al. 2011, A Southern Sky and Galactic Plane Survey for Bright Kuiper Belt Objects, AJ, 142, 98

Spergel, D., Gehrels, N., Breckinridge, J., et al. 2013, Wide-Field InfraRed Survey Telescope-Astrophysics Focused Telescope Assets WFIRST-AFTA Final Report, arXiv: 1305.5422

Spergel, D., Gehrels, N., Breckinridge, J., et al. 2013, WFIRST-2.4: What Every Astronomer Should Know, arXiv:1305.5425 